\title{
神秘的自然常数 $e$
}

\section{吴秋霞 ${ }^{1}$ 卓剑 $^{2}$}

1 宿迁学院 2 宿迁市钟吾国际学校

DOI:10.32629/jief.v2i10.2212

[摘 要] 自然常数 $e$ 跟 $\pi$ 和 $\sqrt{2}$ 相比, 不太容易被学生接受, 因为 $\pi$ 和 $\sqrt{2}$ 都有各自的直观解释: $\pi$ 表示直径为 1 的圆周长, $\sqrt{2}$ 表示边长为 1 的

单位正方形的对角线的长度，本文旨在通过对 1. $e$ 的由来; 2. $e$ 的定义和计算; 这两个方面的阐述，让学生深入了解这个无限不循环小数， 最后通过一个应用, 再次强调 $e$ 的重要性, 并加入课程思政的元素, 旨在教育学生要感恩祖国, 以祖国为荣.

[关键词] 自然常数 $e$; 复利; 自然律; $\lim _{x \rightarrow \infty}\left(1+\frac{1}{n}\right)^{n}=e$; 疫情防控

中图分类号: G633 文献标识码: A

\section{引言}

在《初等数学研究》课程的教学中, 经常会有学生提出类似 “自然 常数 $e$, 我怎么感受不到它的自然呢? ”这样的问题, 基于课程特点, 本文将从 1. $e$ 的由来; $2 . e$ 的定义和计算; 3. 新冠疫情防控中的 $e$ 三个 角度一起探索神秘的自然常数 $e$.

\section{$1 e$ 的由来}

苏格兰数学家约翰. 纳皮尔发明了对数, 人们认为, 纳皮尔对数实际 上就是以 $\frac{1}{e}$ 为底的对数, 但他本人并没有意识到这一点. 1683 年数学家 雅各布. 伯努利提出复利问题, 半个世纪后, 数学大师莱昂哈德. 欧拉在 解决复利问题的过程中, 发现了自然常数 $e$, 因此这个数也被称为欧拉 数.

现在来看复利问题: 所谓复利, 也就是利滚利, 假如在银行存入 1 元钱, 银行给的年利率是 $100 \%$, 一年后你会得到 $(1+1)^{1}=2$ 元的收益（如 图 1), 如果 6 个月结息一次, 一年结算两次, 利率就是 $50 \%$, 那么第 6 个月可以拿到利息 0.5 元, 把这 0.5 元连同本金 1 元一起存入银行, 年后收益为 (如图 2) $(1+0.5)(1+0.5)=\left(1+\frac{1}{e}\right)^{2}=2.25$ 元. 如果每四个月结息一 次且每次结算后利息连同本金立马存入, 一年结算三次, 一年后收益为 $\left(1+\frac{1}{3}\right)^{2} \approx 2.3074$ 元 (如图 3) $\cdots$, 如果每月结息一次呢? 每天呢? 每小时呢? 每分钟、每秒, 甚至每个瞬间呢（假设理论上是可以的）? 你的 1 元钱 会不会变得越来越多, 一年后你就成了大富翁呢? 不可能! 你会发现好 像有一个 “天花板” 挡住了你的发财梦, 这个 “天花板” 是什么呢? 通 过描点作图画出函数 $y=\left(1+\frac{1}{n}\right)^{n}$ 的部分图像, 刚才说的天花板, 就是这条 红线！(如图 4)

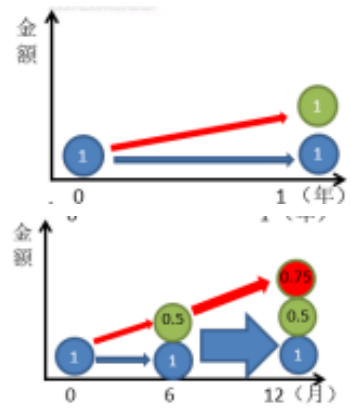

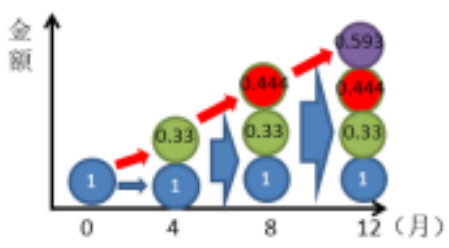

图 3

\section{$2 e$ 的定义和计算}

通过刚才简单的计算和画图: 观察发现红线的位置位于 2.718 到 2.7185 之间. 也就是当 $n \rightarrow \infty$ 时, $\left(1+\frac{1}{n}\right)^{n}$ 可能趋近于一个无限不循环十进小 数, 不妨猜想: $\lim _{x \rightarrow \infty}\left(1+\frac{1}{n}\right)^{n}=e$, 实际上从戴德金分割原理到确界定理, 再到单调有界定理, 我们可以证明 $\lim _{x \rightarrow \infty}\left(1+\frac{1}{n}\right)^{n}$ 极限是存在的, 现在我们来 看一下这个极限值是不是等于 $\mathrm{e}$ 呢?

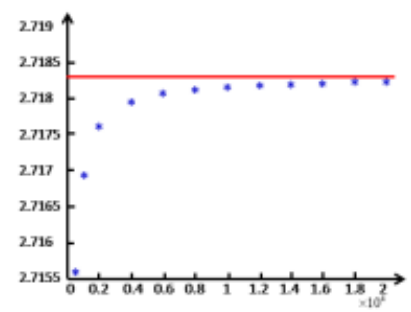

图 4

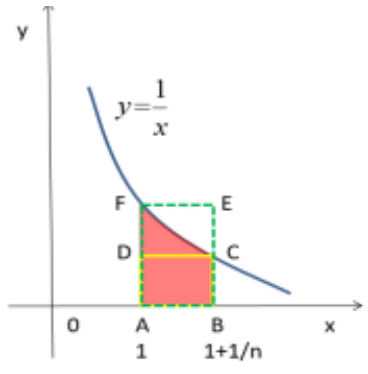

图 5
大家百度搜索，会发现这样一种证明 $\lim _{x \rightarrow \infty}\left(1+\frac{1}{n}\right)^{n}=e$ 的方法: 如图 5 中, 曲边梯形的面积记为 $S$, 则 $\mathrm{S}_{\mathrm{ABCD}}<\mathrm{S}<\mathrm{S}_{\mathrm{ABEF}}$, 其中 $S=\int_{1}^{1+\frac{1}{n}} \frac{1}{x} d x$, 
就有

$\frac{1}{n} \cdot \frac{1}{1+\frac{1}{n}}<1 \frac{1}{1}+\frac{1}{n} \frac{1}{x} d x<\frac{1}{n} \cdot 1$, 化简, 得 $\frac{1}{n+1}<\ln \times \mid \frac{1}{n}<\frac{1}{n}$, 两边同乘以 $n$, 得

$\frac{n}{n+1}<n \cdot \ln \left(1+\frac{1}{n}\right)<1$, 根据两面夹法则, 得 $\lim _{x \rightarrow \infty} \ln \left(1+\frac{1}{n}\right)^{n}=1$,

也就是 $\lim _{x \rightarrow \infty}\left(1+\frac{1}{n}\right)^{n}=e$. 在这个 “证明方法” 中, 涉及了对数函数, 有 “循 环论证”的嫌疑.

实际上我们还是从复利问题着手: 把 $\left(1+\frac{1}{n}\right)^{n}$ 中的 $n$ 取足够大来算算 看！得到下面这个表格:

\begin{tabular}{|l|l|}
\hline$n$ & $\left(1+\frac{1}{n}\right)^{n}$ \\
\hline 1 & 2 \\
\hline 2 & 2.25 \\
\hline 3 & 2.3074 \\
\hline 4 & 2.4414 \\
\hline 5 & 2.4885 \\
\hline 10 & 2.5936 \\
\hline 100 & 2.7051 \\
\hline 1000 & 2.71692 \\
\hline 10000 & 2.71815 \\
\hline
\end{tabular}

可以发现: 要想得到五位准确小数, $n$ 要算到很大, 因为古代没有 计算机, 这对古人来说, 要花费大量时间去计算. 当然我们也可以用牛顿 给出的关于指数函数的幂级数展开式来计算 $e$.

$e^{x}=1+x+\frac{x^{2}}{2 !}+\frac{x^{3}}{3 !}+\frac{x^{4}}{4 !}+\cdots$, 设这个式子中的 $x=1$, 就得到 $e=1+\frac{1}{1 !}+\frac{1}{2 !}+\frac{1}{3 !}+\cdots+\frac{1}{n !}+\cdots$, 用它来计算 $e$ 的值, 请看下表:

\begin{tabular}{|l|l|}
\hline$n$ & $e \approx 1+\frac{1}{1 !}+\frac{1}{2 !}+\frac{1}{3 !}+\cdots+\frac{1}{n !}$ \\
\hline 1 & 2 \\
\hline 2 & 2.5 \\
\hline 3 & 2.666666 \\
\hline 4 & 2.708333 \\
\hline 5 & 2.716666 \\
\hline 6 & 2.718055 \\
\hline 7 & 2.718253 \\
\hline
\end{tabular}

比较两张表, 可以看出用第二种方法比第一种能更快得到准确的 $e$ 的值.

综上所述, $e$ 的含义可以表述为: 单位时间内, 持续的翻倍增长所 接近的极限值. 这个过程体现了 “趋于稳定的平衡” 这个 “自然律” . $e$ 是一个数字, 它本身就是自然存在的, 人类只是发现了 $e$ (不能说证明 了 $e$, 只能说发现了 $e$ ), 因而称之为自然常数. 它的定义式就是 $\lim _{x \rightarrow \infty}\left(1+\frac{1}{n}\right)^{n}=e$.

\section{3 新冠疫情防控中的 $e$}

这场不期而遇的疫情, 让我们看到了中国的强大，更看到了全国人 民的凝聚力. 以 $e$ 为底的自然对数, 在很多领域有着广泛的应用。下面我 们结合中国新冠疫情的防控来看一看它的具体应用!

根据传染病流行的规律, 设 $x$ 是患病人数, $y$ 是接近这些人而被传 染的人数, 它们都是时间 $t$ 的函数, 则有: $\left\{\begin{array}{ll}\frac{d x}{d t}=m x y-n x & \text { (1) } \\ \frac{d y}{d t}=-m x y & \text { (2) }\end{array}\right.$ 其中 $m$ 和 $n$ 分别是不一定相同的正常数，（1）式中的 $m x y$ 表示容易被传染人群中变 成患者的增速, $-n x$ 表示患者因病死而减少的速度, (2) 式中的 $-m x y$ 表 示容易被传染人群中因感染后, 被传染人群减少的速度, (1) + (2) 得 到: $\frac{d x}{d y}=-1+\frac{n}{m y} \quad$ (3) 即 $d x=\left(-1+\frac{n}{m y}\right) d y$ (4) 把 (4) 两边积分, 并代入 初始条件 $\mathrm{x}=\mathrm{x}_{0}, \mathrm{y}=\mathrm{y}_{0}$ 就有: $x=x_{0}+y_{0}-y+\frac{n}{m} \ln \frac{y}{y_{0}} \quad$ (5) 注意! 在预测患者人 数的计算公式 (5) 中出现了 $e$ ! 分析 (3) 式有: 当 $y<\frac{n}{m}$ 时, $\frac{d x}{d y}>0, x$ 是以 $y$ 为自变量的单调递增函数, 当 $y<\frac{n}{m}$ 时, $\frac{d x}{d y}<0, x$ 是以 $y$ 为自变量 的单调递减函数, 这就: 疫情初期即 $y<\frac{n}{m}$ 时, 感染人数增加很快, 应该 加强早期控制, 到了疫情后期, 即 $y<\frac{n}{m}$ 时, 虽然感染人数增加较慢, 但 已经造成了很多人死亡!

由此可见, 我们国家果断封城, 全民动员, 有效进行了早期控制, 尽可能阻止疫情扩散, 最大程度地减少了死亡, 是有科学依据的. 这也反 映了我国政府对生命的敬畏和对科学的尊重.

通过刚才的分析, 我们体会了自然常数 $e$ 的神秘, 感受了它的 “自 然”, 实际上它在很多领域都非常活跃. 更多关于 $e$ 的内容, 期待广大读 者积极去了解和深入研究.

\section{[参考文献]}

[1]王欣,齐新社,王娜.再谈自然常数 e 的存在性及无理性 [J]. 高等数 学研究,2019,22(03):1-2.

[2]李康. 自然常数 $\mathrm{e}$ 的指数形式与生活中物理现象的联系[ J].物理通 报,2019(03):126-128.

[3]庞荣波.浅谈自然常数 $e$ 的命名者一一欧拉 [J].科教文汇(上旬 刊),2009(01):274. 